\title{
PERCEPCIÓN DE LOS ANCIANOS SOBRE LOS CENTROS DE DESARROLLO INTEGRAL PARA EL ADULTO MAYOR
}

\author{
Fernanda Herrera S. ${ }^{1}$, Alehi Romero A. ${ }^{2}$, Emy Gonzales J. ${ }^{3}$ y Leslie Guillen Q. ${ }^{4}$ \\ Universidad Autónoma de Baja California, México \\ (RECIBIDO EL 22/11/2007, ACEPTADO 30/05/2008)
}

\begin{abstract}
RESUMEN
La siguiente investigación pretende explorar el campo de la percepción de los ancianos que acuden al Centro de Desarrollo Integral para el Adulto Mayor en el Municipio de Mexicali (Casa del Abuelo), ubicada en Av. Misión San Diego S/N Fracc. Hacienda Dorada. La metodología utilizada consistió en entrevistas semiestructuradas y grabaciones de audio, que posteriormente se analizaron bajo la perspectiva fenomenológica de los casos estudiados. La finalidad de la misma pretende, al exponer los casos, contribuir al cambio de la percepción cultural sobre los mismos, conocer los factores externos e internos que afectan el desarrollo pleno en esta etapa de la vida, así como reconocer la importancia que tiene la creación de lugares especializados para contribuir al cuidado bio-psico-social de los individuos.
\end{abstract}

Palabras clave: Percepción, Factores externos, Factores internos, Concepción cultural, Depresión.

\begin{abstract}
The following investigation tries to explore the field of the perception of the old ones, which they go to the centers of integral development for the old people in the municipality of Mexicali; the methodology used consisted of semi structured interviews and audio recordings, which later they were analyzed under the phenomenological perspective of the studied cases. the purpose of the same one, tries by exposing the cases, to contribute to the change of the cultural perception on such, to know external and internal the factors that affect the total development in this stage of the life, as well as, to recognize the importance that has the creation of specialized places in contributing to the bio-psico-social care of the individuals.
\end{abstract}

Keywords: Perception, External factors, Internal factors, Cultural conception, Depression.

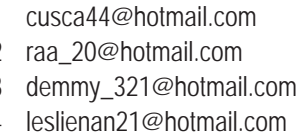




\section{INTRODUCCIÓN}

El significado de edad adulta mayor o tardía ha cambiado en los últimos tiempos, ya que es reciente la relevancia que ha tomado el estudio de esta etapa de la vida del ser humano, que antes estaba aislada de las investigaciones o faltaba interés en ella. En la actualidad, la longevidad no tiene parámetros muy definidos, la edad adulta mayor o tardía comienza su periodo a partir de los 65 años, sin embargo adultos mayores de 75 u 85 años de edad refieren no sentirse viejos. Esto se debe, probablemente, a las influencias en los periodos del desarrollo tales como la herencia, el ambiente y la maduración, así como a las influencias del contexto donde se desarrolló el individuo, marcando una diferencia individual esencial en cómo afrontar la llegada de la vejez. El funcionamiento físico y cognoscitivo determinarán en gran medida el estado emocional de la persona, así como su percepción de ser capaz de llevar una vida con cierta independencia. Los enfoques sobre esta etapa de la vida varían según los autores; para Erikson, el mayor logro de esta etapa es la integridad del yo, basado en la reflexión acerca de la propia vida. Esta etapa está limitada tanto por condiciones objetivas externas al individuo como por condiciones subjetivas del mismo, además de los procesos biológicos vinculados a ésta. Será la suma de la adaptación de estos factores lo que disponga o predisponga al individuo a vivir una vejez programada, planificada y aceptada o una vejez resultante de las frustraciones de toda una vida; el conocimiento de estos factores puede orientarnos para facilitar o favorecer la integración de las personas en esta etapa a un ambiente donde se sepan reconfortados y encuentren nuevos significados personales a su vida para la aceptación y estima de sí mismos.

El propósito de la siguiente investigación será explorar aquellos factores que influyen en la percepción que tiene el adulto mayor sobre sí mismo, en la etapa de vida que están cursando y sobre su estancia en los centros de desarrollo para el adulto mayor como un fenómeno cada vez más común, usando como base o guía una entrevista semi-estructurada.

\section{Antecedentes teóricos}

Desde el inicio de los tiempos, el ser humano se ha interesado en los cambios fisiológicos y mentales que sufre el hombre a lo largo del ciclo de la vida; desde Aristóteles, que se enfocaba en los aspectos somáticos del envejecimiento, a Da Vinci, se enfocaba en los cambios anatómicos del cuerpo; sin embargo, es recién en el siglo XIX cuando empieza un impulso a la gerontología, definida como la ciencia que estudia el envejecimiento en todos sus aspectos, e incluye las ciencias biológicas y médicas, psicológicas y sociológicas; además de la aplicación del conocimiento científico en beneficio del envejecimiento y de los adultos mayores.

Erikson creía que, aunque las funciones del cuerpo a esta edad se debilitaban, la gente debía mantener cierto compromiso con la sociedad, que la integridad de las personas se basaba en las reflexiones que éstas hacían sobre su vida. Es en esta etapa cuando, por lo general, surge un distanciamiento social gradual, a veces provocado por su sentimiento de inutilidad o frustración debido a que ya no pueden realizar actividades que antes acostumbraban o simplemente porque su vida laboral termina a esta edad aproximadamente. Junto con la aparición de enfermedades relacionadas al proceso de envejecimiento, surgen temores 
nuevos, como el sentirse cada vez más cercanos a la muerte, a la soledad, a la viudez; muchos empiezan a perder amigos de toda la vida, a sus cónyuges e incluso a sus mismos hijos. El comportamiento en la vejez lo entendemos como resultado del paso del tiempo sobre el organismo y que éste afecta determinadas características tanto de la personalidad como del funcionamiento intelectual, etc. En general, lo apreciamos como un decremento en todas las funciones y habilidades del sujeto, aun más cuando la cultura que nos envuelve es la de la juventud, donde no hay cabida para la sabiduría de los ancianos, donde estos quedan relegados a desempeñar un restringido campo de actividades que ofrecen pocas motivaciones al adulto mayor.

Existe una teoría que nos habla de que es el mismo anciano quien busca este distanciamiento al ver su propio declive funcional y que es un proceso normal en todos los individuos. Esta teoría llamada Teoría del Desapego (Cummings y Henry) sugiere que el sujeto que envejece va perdiendo interés vital por los objetos y actividades que le posibilitan una interacción social y se produce una apatía emocional sobre los otros y, al mismo tiempo, el sujeto se encierra en sus propios problemas.

Todos estos factores pueden verse afectados también en gran medida mediante la percepción del individuo sobre su propia existencia, y hablar de la percepción involucra un mundo derivado de los sentidos en los que el proceso en cada uno de los individuos será diferente. El proceso de la percepción, tal como propuso Hermann von Helmholtz, es de carácter inferencial y constructivo, generando una representación interna de lo que sucede en el exterior a modo de hipótesis. Para ello se usa la información que llega a los receptores y se va analizando, paulatinamente, la información que viene de la memoria, tanto empírica como genética, y que ayuda a la interpretación y a la formación de la representación.

Mediante la percepción, la información recopilada por todos los sentidos se procesa y se forma la idea de un solo objeto. Es posible sentir distintas cualidades de un mismo objeto y, mediante la percepción, unirlas, determinar de qué objeto provienen y determinar, a su vez, que es un único objeto. Así entonces puede ser que una persona viviendo experiencias similares a otra, tenga una visión totalmente distinta sobre la misma porque su proceso de percepción es producto de su interpretación personal. La percepción será, entonces, un factor intrínseco o interno que afecte directamente la vivencia de la vejez de los sujetos.

También hay algunas teorías que nos hablan sobre los factores psicosociales del envejecimiento, como las siguientes:

\section{Teorías psicosociales del envejecimiento}

Teoría de la Separación. Afirma que a medida que la gente va envejeciendo, aparece una tendencia natural a alejarse social y psicológicamente del ambiente, de las actividades sociales y de otras personas.

Teoría de la Actividad. Sugiere que la continuación de un estilo de vida activo tiene un efecto positivo sobre la sensación de bienestar y satisfacción de los viejos.

Teoría de la Personalidad y el estilo de vida. Demuestra que existe una relación entre el tipo de personalidad y los patrones de envejecimiento. 
Teoría de Intercambio. Sostiene que las personas con las mayores necesidades son las que pierden más poder y que quienes satisfacen sus necesidades son las que obtienen el poder.

Teoría de la Reconstrucción Social. Describe la manera en que la sociedad reduce el autoconcepto de los ancianos y propone formas de revertir este ciclo negativo.

Los individuos interpretan situaciones cotidianas de distinta forma según el papel que interpretan dentro de la sociedad, estas situaciones o influencias ambientales abarcan los campos de empleo, hogar, relaciones y, si alguna de éstas no es satisfactoria, hará que la persona se frustre y llegue a caer en depresión"; si estas esperanzas, aspiraciones y expectativas se ven defraudadas constantemente el sujeto corre gran peligro" (Bretón, 1996).

Uno de los problemas más comunes es la depresión profunda, que se caracteriza porque las personas mayores sufren pérdidas acumulativas, por ejemplo la muerte de cónyuges y amigos. El deterioro de su salud, capacidad física (lo que las hace sentirse menos independientes), y la pérdida de control contribuyen a la persistencia de la depresión (Penninx et al., 1998; Kahn, Hessling y Russell, 2003).

Esas explicaciones tienen sentido, pero no es del todo claro que la depresión sea un problema significativamente peor en la adultez tardía que más temprano en la vida. Algunos estudios sugieren que la tasa de depresión en realidad sería menor durante la edad adulta tardía. Una razón para este hallazgo contradictorio es que existen dos tipos de depresión, una que continúa desde las etapas tempranas de la vida y la otra que ocurre como resultado del envejecimiento (Gatz, 1997).

La concepción del anciano difiere culturalmente, en algunas organizaciones sociales son respetados e incluso son los líderes de las mismas, en otras se deshacen de ellos como si fueran objetos inservibles. Estas variables no están constituidas al azar, más bien obedecen a razones históricas, económicas, religiosas, antropológicas, políticas e incluso geográficas. En la cultura occidental puede observarse la figura del abuelo en dos puntos extremos, o es idealizado o despreciado (Solís-Ponton, 2004).

Para muchos países, el incremento de la esperanza de vida supone el aumento en problemas de salud, sociales, económicos y políticos; por ello la ONU, en el año 1982, convocó a los países miembros a trabajar en la evaluación y solución de los problemas asociados al envejecimiento (Correa, Vargas y Barrios, 1993).

Toda la información que recibimos culturalmente sobre cómo es la vejez, sus procesos fisiológicos, mentales, sociales etc., en sí, el fenómeno vejez en gran parte objetivo se ve truncado cuando descubrimos que este fenómeno tiende a ser más subjetivo y que cada individuo, pese a la carga social, emocional y psicológica, tendrá una percepción distinta de su propio proceso, independiente de las interpretaciones generales. Los siguientes casos expuestos son un elemento clave de esta investigación, ya que la percepción de los individuos se ve reflejada en los mismos. 


\section{MÉTODO}

\section{Caso 1}

Mujer de 65 años, nacida en Sinaloa que vino a residir a esta capital cuando se casó con un hombre 15 años mayor que ella, a la edad de 20 años, no tuvo escolaridad alguna por lo que su vida laboral se vio restringida a la venta de comidas y limpieza de casas, así como al cuidado de niños. Su relación matrimonial fue siempre mala, el marido la celaba intensamente, la encerraba y no la dejaba salir de casa; era mujeriego y ella sabia de múltiples mujeres con las que el también tenía hijos. La economía familiar era mala ya que el marido no aportaba dinero, por eso ella a escondidas se dedicó a trabajar en horarios en que el marido no estaba en casa para no tener problemas. El marido era alcohólico por lo que ella decidió alejarse de él, pero este siempre la encontraba. Refirió que en varias ocasiones la obligó a sostener relaciones sexuales y que dos de sus hijas son producto de éstas. En la actualidad la relación con sus hijos es mala ya que estos no la visitan y le reprochan la ausencia del padre y de ella misma por tener que salir a trabajar; vive sola y por esta razón decidió buscar un lugar donde estuviera acompañada, ya que no cuenta con buena salud, pues padece de las rodillas y de la columna, y necesita cuidados especiales. Inferimos que se siente satisfecha de su vida ya que no logró que varios aspectos de ésta se llevaran a cabo como ella hubiese deseado.

Podemos concluir que todos los factores externos contribuyeron a la percepción que tiene de su actual situación; sumado a los aspectos intrínsecos como la salud que limitan sus facultades físicas y la aíslan de la con vivencia social.

\section{Caso 2}

Isidro es un señor de 81 años de edad, viudo desde hace 5 años, y jubilado de las oficinas del ISSTE desde hace 28 años. Tiene 3 hijos, dos hombres y una mujer; dos de ellos viven en Mexicali, el otro vive en México.

Él vive en la casa de su hija, con su yerno y nietos desde hace dos años, pero le construyeron un lugar aparte con todos los servicios, se lleva muy bien con toda su familia, tiene 7 nietos. Comentó que extraña mucho su casa de México, pero que sabe que no puede estar allá porque estaría solo y no habría quién lo acompañara.

Él se enteró de la Casa del Abuelo, gracias a una amiga de su hija que trabaja en el DIF, pero antes de ingresar le propusieron esta opción, él asiste porque la vida de aquí es muy diferente a la que llevaba en México, allá él tenia más libertad de salir a cualquier lado en cambio aquí no, además también asiste para no sentirse solo, ya que sus hijos se van a trabajar y sus nietos a la escuela. Comenta que él se siente muy bien en este lugar, que el servicio que recibe es muy bueno, la atención por parte del personal es de calidad, además que tiene ciertas actividades, se la pasa muy bien y no tiene ninguna queja ya que se lleva muy bien con todos en el centro.

Dice que esta etapa de su vida le ha costado mucho trabajo, primero por el cambio de ciudad y el haberse quedado solo, pero sabe que no queda otra cosa más que adaptarse a la vida. Él quisiera que su esposa todavía estuviera con él, porque llevaban una vida muy bonita. 
Hace 3 meses le hicieron unos estudios y salió que tenia un problema en la próstata, los doctores le dicen que tiene que estar en tratamiento y que con eso se corregirá el problema, que lleva su tiempo pero que saldrá adelante, con esta noticia se sintió mal pero que ya está mejor, y otra cosa buena es que no tiene dolor por esto.

\section{DISCUSIÓN}

Los autores revisados concuerdan, al igual que la investigación realizada acerca de la percepción de los ancianos sobre los centros de desarrollo integral del adulto mayor, en que el estilo de vida influirá en la manera en que éste experimente positiva o negativamente su etapa de senectud; sin embargo, es importante tomar en cuenta de que la percepción es un proceso individual y aislado de cada sujeto, ya que es un proceso subjetivo que no se apega a reglas generales.

\section{CONCLUSIONES}

La teoría nos habla de múltiples aspectos y procesos que afectan el desarrollo de la personalidad, así, ésta se verá reflejada en las actitudes del sujeto frente a las distintas situaciones que habrá de afrontar. La etapa de la vejez o del adulto mayor es un resultado de todas las experiencias a lo largo de la vida, por eso es crucial, en la percepción del individuo sobre ésta, todas aquellas etapas anteriores y la manera en que fueron afrontadas. Los periodos de la infancia, el desarrollo psicosocial del sujeto, así como las relaciones familiares, emocionales, el desarrollo laboral, académico. En el desarrollo de la presente investigación fue evidente que el sujeto que se sentía más conforme con su situación actual de vida, era aquel que había desarrollado más los aspectos familiares, emocionales y laborales; a pesar de que ya afrontaba pérdidas del cónyuge, la percepción de la etapa que está viviendo es de satisfacción, al contrario del sujeto que había tenido experiencias desagradables en el ámbito emocional conyugal y malas relaciones familiares. Por lo tanto, podemos concluir que es indispensable fomentar en los adultos mayores espacios donde puedan recrearse y estimular sus habilidades sociales y creativas, para volver a incluir dentro del ámbito social a aquellos que se encuentren aislados y para seguir estimulando a aquellos que han seguido con su actividad laboral hasta su jubilación.

\section{RECOMENDACIONES}

$\mathrm{Al}$ ser una de las limitaciones de esta investigación el campo reducido donde se llevó a cabo, una de las recomendaciones es que se realice de manera más amplia en diversos centros de desarrollo para el adulto mayor, para así obtener resultados más homogéneos sobre las muestras del mismo. 


\section{ANEXOS}

Sujeto 1

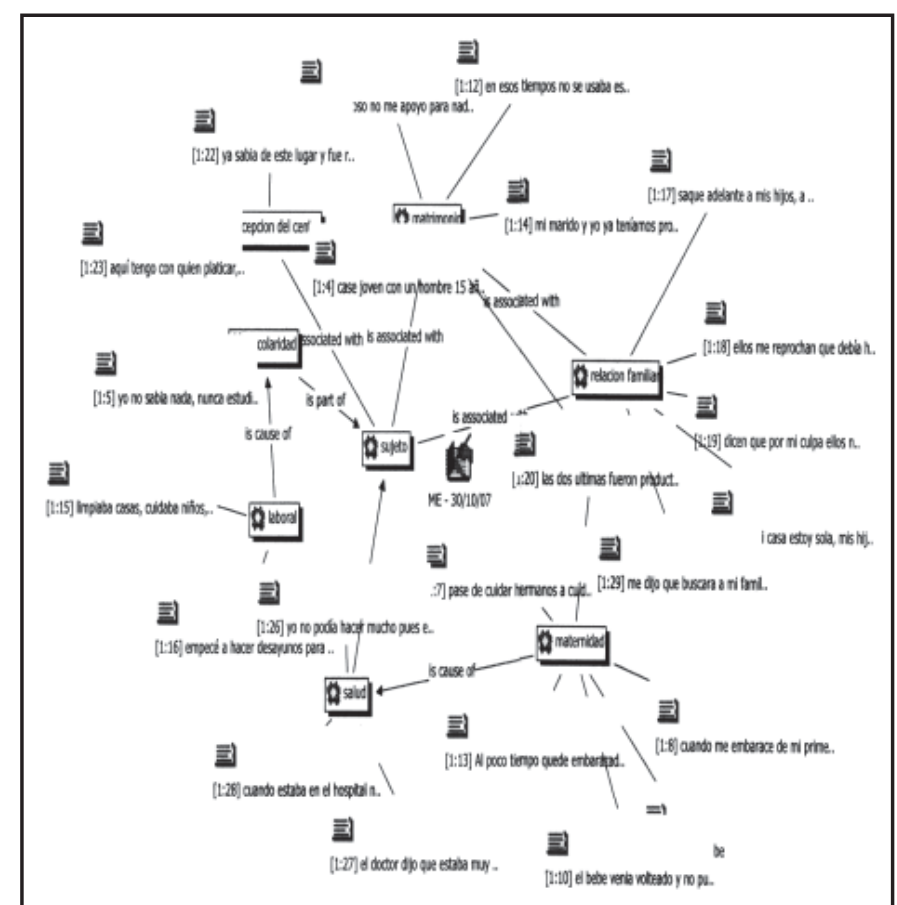

Sujeto 2

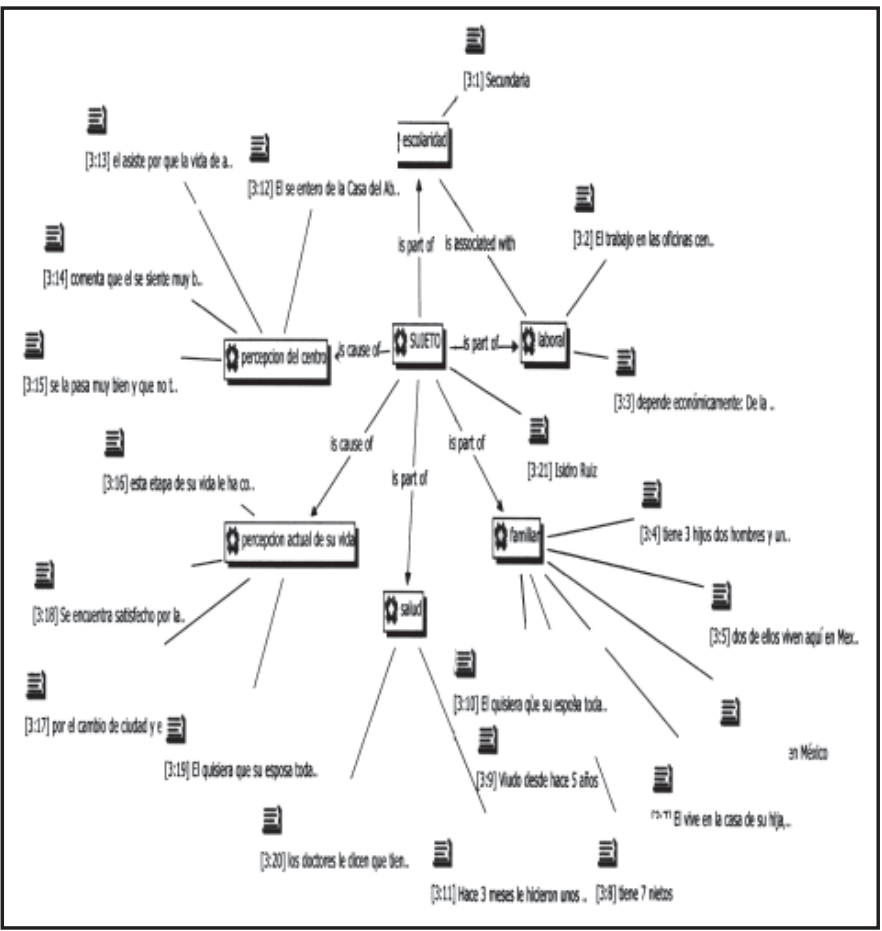

Revista de Investigación en Psicología - Vol. 11, № 1 


\section{REFERENCIAS BIBLIOGRÁFICAS}

1. Cummings y Henry (1961). El proceso de desapego. New York: Pub.

2. Yepes R., Aranguren J. (1999.). Fundamentos de antropología. Una idea de la excelencia humana. $4 .{ }^{a}$ edic. Editorial EUNSA. http://www.psicologia-online.com/ monografias/4/index.shtml

3. Philipe Rice, F. (1997). Desarrollo humano. Estudio del ciclo vital. 4. ${ }^{a}$ edic., Prentice Hall.

4. Breton Sue (1996). La depresión. España: Edit. Martínez Roca, S.A.

5. Vera Noriega, J.A.; Sotelo Quiñones, T. I. y Domínguez Guedea, M.T (2005), Revista intercontinental de psicología y educación vol. $7 \mathrm{~N} .^{\circ}$ 2, julio-diciembre 2005. Edit. Universidad Intercontinental Bibliografía.

6. Robert S. Feldman (2007). Desarrollo psicológico a través de la vida. $4 .{ }^{a}$ edic. Person Prentice Hall.

7. Grace J. Craig (1994). Desarrollo psicológico. Prentice Hall. 